\title{
Variação da Cor da Madeira de Teca em Função da Densidade e do Teor de Extrativos
}

\author{
Rosilei Aparecida Garcia ${ }^{1}$, Gabriel Bittencourt Marinonio ${ }^{1}$
}

${ }^{1}$ Departamento de Produtos Florestais, Universidade Federal Rural do Rio de Janeiro - UFRRJ, Rio de Janeiro/RJ, Brasil

\section{RESUMO}

O objetivo deste estudo foi avaliar o efeito da densidade e do teor de extrativos na variação da cor da madeira de teca (Tectona grandis L. f.) proveniente de plantios de diferentes espaçamentos. As análises colorimétricas foram realizadas nas faces radial e tangencial das madeiras de cerne e alburno com o espectrofotômetro CM 2600d no espaço de cor $\mathrm{L}^{*} \mathrm{a}^{*} \mathrm{~b}^{*}$. A densidade e o teor de extrativos foram determinados segundo as normas ASTM D2395 e ASTM D1105. A madeira do maior espaçamento de plantio apresentou maior densidade e maior teor de extrativos. Na seção radial, as madeiras de cerne de maior densidade apresentaram mais pigmento amarelo, enquanto que na seção tangencial elas são mais escuras e apresentam menos pigmento amarelo. As madeiras de alburno de maior densidade são mais escuras e apresentam mais pigmento vermelho em ambas as seções. As madeiras mais escuras e com mais pigmento vermelho apresentaram um maior teor de extrativos.

Palavras-chave: colorimetria, qualidade da madeira, sistema CIE L*a $\mathrm{a}^{\star} \mathrm{b}^{\star}$.

\section{Color Variation of the Teak Wood as a Function of Density and Extractive Content}

\begin{abstract}
The objective of this study was to evaluate the effect of density and extractive content on color variation of teak (Tectona grandis L. f.) wood from plantation of different tree-spacing. Color analyses were performed on radial and tangential sections of heartwood and sapwood by using a CM 2600d spectrophotometer on the $\mathrm{L}^{*} \mathrm{a}^{\star} \mathrm{b}^{*}$ color space. Density and extractive content were determined following the ASTM D2395 and ASTM D1105 standards. The wood from larger tree-spacing had higher density and extractive content. In the radial section, denser heartwoods had more yellow hue, while in tangential section, they are darker and had less yellow hue. Denser sapwoods are darker and had more red hue in both sections. Darker woods and with more red hue presented higher extractive content.
\end{abstract}

Keywords: colorimetry, wood quality, CIE $\mathrm{L}^{\star} \mathrm{a}^{\star} \mathrm{b}^{\star}$ system. 


\section{INTRODUÇÃO}

A teca (Tectona grandis L. f., Família Verbenaceae) é uma das mais valiosas e mais importantes espécies tropicais no mercado internacional devido à excelente qualidade de sua madeira e ao ciclo de rotação relativamente curto situado entre 20 e 25 anos (Premrasmi \& Dietrichs, 1967; Schmincke, 2000; Moya \& Pérez, 2007; Derkyi et al., 2009; Miranda et al., 2011).

A madeira de teca é moderadamente dura e pesada, com densidade variando entre 0,44 e 0,82 g. $\mathrm{cm}^{-3}$, apresenta bom comportamento na secagem e boa trabalhabilidade, sendo utilizada em estruturas internas e externas, na confecção de lâminas, na fabricação de compensados, no mobiliário fino, na construção naval e na carpintaria em geral (Premrasmi \& Dietrichs, 1967; Baillères \& Durand, 2000; Miranda et al., 2011). O cerne apresenta alta durabilidade natural e alta estabilidade dimensional, características devidas à presença de algumas substâncias, como o caucho, espécie de látex responsável pela redução de absorção de água, e a tectoquinona, um preservativo natural contido nas células da madeira (Narayanamurti \& Singh, 1960; Premrasmi \& Dietrichs, 1967; Yamamoto et al., 1998; Kokutse et al., 2006).

A qualidade da madeira é geralmente determinada pelas suas propriedades físicas e mecânicas, sendo características como densidade, dureza, textura e grã bastante utilizadas na classificação e na avaliação da qualidade (Mori et al., 2005). Entretanto, características estéticas como cor e desenho são atributos importantes por direcionarem o uso da madeira para suas diferentes finalidades e estabelecerem o seu valor comercial (Janin et al., 2001). Möttönen et al. (2002) também observaram que as características e a uniformidade da cor da madeira têm um papel direto no estabelecimento do preço e no valor final do produto. Por essa razão, a cor também é considerada importante índice de classificação e qualidade, devendo ser incorporada na caracterização tecnológica da madeira, visando atender aos usos mais nobres desse material (Mori et al., 2004, 2005)

A cor natural da madeira pode ser influenciada por fatores genéticos e ambientais como espécie; composição química (principalmente teor e natureza dos extrativos); estrutura anatômica (anéis de crescimento, vasos, raios e tipo de parênquima axial); posição de amostragem na árvore (nos sentidos radial e axial); condições edáficas e climáticas; altura, diâmetro e idade da árvore; tratos silviculturais; e taxa de crescimento da árvore (McGinnes \& Phelps, 1983; Desch \& Dinwoodie, 1993; Gonçalez, 1993; Mady, 2000; Kokutse et al., 2006; Derkyi et al., 2009).

A madeira de teca é porosa a semiporosa com anéis de crescimento distintos demarcados por linhas de parênquima marginal, com alburno claro, bem distinto do cerne, cuja cor é marrom viva e brilhante. Segundo Tsoumis (1968), a variação da cor natural da madeira deve-se à impregnação de diversas substâncias orgânicas nas paredes celulares, depositadas de forma mais acentuada no cerne.

Devido à importância da cor da madeira e à sua grande variabilidade, estudos sobre o efeito dos tratamentos silviculturais e das características daqueles materiais na formação da cor são necessários, visando determinar as condições mais adequadas de cultivo para a obtenção de produtos de qualidade. Nesse contexto, o objetivo deste trabalho foi avaliar o efeito da densidade e do teor de extrativos na variação da cor da madeira de teca proveniente de plantios com diferentes espaçamentos.

\section{MATERIAL E MÉTODOS}

\subsection{Material}

Foram utilizadas duas árvores de teca (Tectona grandis L. f.) de 12 anos de idade, provenientes de plantios de dois diferentes espaçamentos $(5 \times 2 \mathrm{~m} \mathrm{e} 6 \times 2 \mathrm{~m})$ localizados na zona experimental do Instituto Federal de Educação, Ciência e Tecnologia de Mato Grosso em Cáceres, MG, coordenadas geográficas $16^{\circ} 11^{\prime} 42^{\prime \prime} \mathrm{S}$ e $57^{\circ} 40^{\prime} 51^{\prime \prime} \mathrm{W}$ e altitude de $117 \mathrm{~m}$. Discos de aproximadamente $50 \mathrm{~mm}$ de espessura foram retirados à $2 \mathrm{~m}$ de altura à partir do DAP e então amostras de cerne e alburno, com dimensões de $50 \times 25 \times 25 \mathrm{~mm}$ (comprimento $\times$ largura $\times$ espessura) e perfeitamente orientadas nos planos transversal, radial e tangencial foram obtidas. As amostras foram acondicionadas em câmara climática a $20{ }^{\circ} \mathrm{C}$ e $65 \%$ de umidade relativa até obtenção de peso constante.

A densidade aparente da madeira foi determinada de acordo com a norma ASTM D 2.395 (ASTM, 1999a). 


\subsection{Análises colorimétricas no espaço de cor $L^{*} a^{\star} b^{\star}$}

As análises colorimétricas foram realizadas com o auxílio do espectrofotômetro portátil CM $2600 \mathrm{~d}$ da Konica Minolta no espaço de cor L $\mathrm{L}^{\star} \mathrm{a}^{\star} \mathrm{b}^{\star}$ CIE 1976, segundo a norma ISO 11.664-4:2008 (ISO, 2008), o qual é caracterizado por três coordenadas $\left(\mathrm{L}^{*}, \mathrm{a}^{*} \mathrm{e} \mathrm{b}^{*}\right) \mathrm{em}$ um espaço tridimensional. A coordenada $\mathrm{L}^{\star}$ representa a luminosidade ou claridade, a qual varia de zero (preto) a 100 (branco), enquanto que $\mathrm{a}^{\star}$ e $\mathrm{b}^{\star}$ representam as variáveis cromáticas nos eixos verde-vermelho e azul-amarelo, respectivamente, ambas variando entre -60 e +60 . Os sinais positivo e negativo indicam o aumento na tonalidade: vermelha $\left(+\mathrm{a}^{*}\right)$, verde $\left(-\mathrm{a}^{*}\right)$, amarela $\left(+b^{\star}\right)$ e azul $\left(-b^{\star}\right)$ (Konica Minolta, 1998).

A medição foi realizada através do contato direto da superfície da amostra, em pontos pré-determinados, e a área de iluminação do aparelho, com diâmetro de $3 \mathrm{~mm}$ (SAV - Small Area View), utilizando-se iluminante padrão $\mathrm{D}_{65}$ e observador padrão suplementar $10^{\circ}$. Antes das medições, a superfície das amostras foi polidas com uma sequência de lixas de granulometria 120 e 220 .

Para cada amostra foram determinadas as variáveis $L^{*}, a^{*}$ e $b^{*}$ nas seções radial $\left(L^{*}\right.$-radial, $a^{*}$-radial e $\mathrm{b}^{\star}$-radial) e tangencial ( $\mathrm{L}^{\star}$-tang, $\mathrm{a}^{\star}$-tang e $\mathrm{b}^{\star}$-tang). Foram efetuadas cinco observações em cada seção de cada amostra, para um total das 20 amostras, totalizando 200 observações.

Os dados coletados pelo espectrofotômetro foram então transferidos para o software SpectraMagic $\mathrm{NX}^{\mathrm{TM}}$ (Konica Minolta) e então exportados para o software Microsoft Office Excel.

\subsection{Determinação do teor de extrativos}

O teor de extrativos da madeira foi determinado de acordo com o método de análises químicas descrito por Abreu et al. (2006), o qual foi adaptado a partir da norma ASTM D 1.105 (ASTM, 1999b). Para tal análise, as mesmas amostras utilizadas para determinação da densidade e da cor foram fragmentadas e posteriormente moídas em um moinho de rotor vertical modelo MA 340 da Marconi ${ }^{\oplus}$, seguindo as especificações da norma ABTCP M1/71 (ABTCP, 1971). A extração foi realizada a partir de 20 gramas da amostra seca ao ar com três solventes orgânicos de ordem crescente de polaridade: ciclohexano, acetato de etila e metanol, utilizando-se um extrator Soxhlet durante 24 horas ininterruptas com cada solvente. Após cada extração, utilizou-se um rotavapor giratório para retirar o excesso de solvente por sucção e os extrativos foram armazenados em recipientes de vidro previamente tarados e depositados em capela para a completa evaporação do solvente. Em seguida, os recipientes de vidro contendo os extrativos foram pesados, obtendo-se a massa de extrativos em gramas. Considerando-se a quantidade de partículas empregadas, calculou-se a porcentagem de extrativos de acordo com a norma ABTCP M2/71 (ABTCP, 1971). Foi realizada somente uma repetição para cada tratamento.

\subsection{Análises estatísticas}

Os dados obtidos para a densidade aparente e as coordenadas de cor $\left(L^{*}, a^{*} e b^{*}\right)$ foram submetidos à uma análise de variância com o auxílio do programa SAS $^{\star}$ System. Quando o valor $\mathrm{F}$ foi significativo $(\alpha=0,05)$, aplicou-se o teste de Duncan $(\alpha=0,05)$ a fim de determinar as diferenças significativas entre as médias de cada tratamento. As variáveis independentes estudadas foram: espaçamento $(6 \times 2 \mathrm{~m}$ e $5 \times 2 \mathrm{~m})$ e tipo de madeira (cerne e alburno), enquanto que as variáves dependentes foram: densidade, $L^{*}$-radial, $\mathrm{a}^{\star}$-radial, $\mathrm{b}^{\star}$-radial, $\mathrm{L}^{*}$-tang, $\mathrm{a}^{*}$-tang, $\mathrm{b}^{\star}$-tang e teor de extrativos.

Análises de correlação de Pearson foram realizadas a fim de determinar o efeito da densidade e do teor de extrativos na variação da cor da madeira de cerne e alburno.

\section{RESULTADOS E DISCUSSÃO}

\subsection{Efeito do espaçamento na variação da densidade e da cor da madeira}

As análises estatísticas indicaram diferenças significativas entre as densidades da madeira dos dois espaçamentos (Tabela 1). As madeiras do maior espaçamento $(6 \times 2 \mathrm{~m})$ apresentaram densidades mais altas que aquelas do menor espaçamento $(5 \times 2 \mathrm{~m})$. A densidade da madeira representa a quantidade de massa por unidade de volume e está relacionada às características anatômicas como diâmetro e frequência dos poros, espessura de parede das fibras, proporção de lenho tardio e inicial, largura dos anéis de crescimento, 
Tabela 1. Estatística descritiva (média e coeficiente de variação) e valores de F obtidos pela análise de variância para densidade aparente e coordenadas colorimétricas da madeira de teca proveniente de diferentes espaçamentos. Table 1. Descriptive statistical analysis (means and coefficient of variation) and F values obtained by analysis of variance for density and color coordinates of teak wood from different spacing.

\begin{tabular}{|c|c|c|c|c|c|}
\hline \multirow{2}{*}{ Variáveis } & \multicolumn{2}{|c|}{ Espaçamento $6 \times 2 \mathrm{~m}$} & \multicolumn{2}{|c|}{ Espaçamento $5 \times 2 \mathrm{~m}$} & \multirow{2}{*}{ Valor F } \\
\hline & Cerne & Alburno & Cerne & Alburno & \\
\hline $\begin{array}{c}\text { Densidade } \\
\left(\mathrm{g} \mathrm{cm}^{-3}\right)\end{array}$ & $\begin{array}{c}0,607 \mathrm{~b} \\
{[1,65]}\end{array}$ & $\begin{array}{c}0,627 \mathrm{a} \\
{[1,59]}\end{array}$ & $\begin{array}{c}0,585 \mathrm{c} \\
{[1,71]}\end{array}$ & $\begin{array}{c}0,562 \mathrm{~d} \\
{[1,78]}\end{array}$ & $25,62^{* *}$ \\
\hline $\mathrm{L}^{*}$-radial & $\begin{array}{c}59,17 \mathrm{c} \\
{[5,07]}\end{array}$ & $\begin{array}{c}72,19 \mathrm{~b} \\
{[2,84]}\end{array}$ & $\begin{array}{c}59,47 \mathrm{c} \\
{[5,60]}\end{array}$ & $\begin{array}{c}77,60 \mathrm{a} \\
{[1,75]}\end{array}$ & $26,56^{\star *}$ \\
\hline $\mathrm{a}^{*}$-radial & $\begin{array}{c}10,42 \mathrm{a} \\
{[4,80]}\end{array}$ & $\begin{array}{l}4,26 \mathrm{c} \\
{[7,98]}\end{array}$ & $\begin{array}{l}9,25 \mathrm{~b} \\
{[5,30]}\end{array}$ & $\begin{array}{l}3,36 \mathrm{~d} \\
{[5,95]}\end{array}$ & $125,67^{\star *}$ \\
\hline $\mathrm{b}^{*}$-radial & $\begin{array}{c}26,78 \mathrm{a} \\
{[3,02]}\end{array}$ & $\begin{array}{c}19,10 \mathrm{c} \\
{[4,60]}\end{array}$ & $\begin{array}{l}21,51 \mathrm{~b} \\
{[11,62]}\end{array}$ & $\begin{array}{c}18,43 \mathrm{c} \\
{[4,50]}\end{array}$ & $18,64^{\star *}$ \\
\hline $\mathrm{L}^{*}$-tang & $\begin{array}{c}61,27 c \\
{[1,48]}\end{array}$ & $\begin{array}{c}69,52 \mathrm{a} \\
{[2,12]}\end{array}$ & $\begin{array}{c}65,78 \mathrm{~b} \\
{[2,40]}\end{array}$ & $\begin{array}{c}71,50 \mathrm{a} \\
{[1,12]}\end{array}$ & $19,21^{\star *}$ \\
\hline $\mathrm{a}^{\star}$-tang & $\begin{array}{c}9,52 \mathrm{a} \\
{[10,19]}\end{array}$ & $\begin{array}{l}5,34 \mathrm{c} \\
{[3,74]}\end{array}$ & $\begin{array}{l}8,65 \mathrm{~b} \\
{[5,66]}\end{array}$ & $\begin{array}{l}5,17 \mathrm{c} \\
{[4,06]}\end{array}$ & $39,45^{\star *}$ \\
\hline $\mathrm{b}^{*}$-tang & $\begin{array}{c}25,94 \mathrm{a} \\
{[7,21]}\end{array}$ & $\begin{array}{c}20,74 \mathrm{~b} \\
{[1,83]}\end{array}$ & $\begin{array}{c}27,03 \mathrm{a} \\
{[6,73]}\end{array}$ & $\begin{array}{c}21,43 \mathrm{~b} \\
{[2,52]}\end{array}$ & $13,17^{\star *}$ \\
\hline
\end{tabular}

$\mathrm{L}^{*}$-radial e $\mathrm{L}^{*}$-tang $=$ luminosidade das seções radial e tangencial, respectivamente; $\mathrm{a}^{*}$-radial e $\mathrm{a}^{*}$-tang $=$ variável cromática $\mathrm{a}^{*}$ (vermelho) nas seções radial e tangencial, respectivamente; $b^{\star}$-radial e $b^{\star}$-tang $=$ variável cromática b* (amarelo) nas seções radial e tangencial, respectivamente; Valores entre colchetes = coeficiente de variação experimental. ${ }^{\star *}$ Significativo à $99 \%$ de probabilidade. Médias com a mesma letra não diferem estatisticamente entre si segundo o teste de Duncan.

entre outras (Panshin \& Zeeuw, 1980). A madeira de teca é semiporosa, sendo o lenho inicial composto por vasos de grande diâmetro com grande volume de vazio e o lenho tardio composto por maior quantidade de material lenhoso, ou seja, com maior quantidade de fibras com paredes espessas e vasos de menor diâmetro. A madeira de teca proveniente do maior espaçamento $(6 \times 2 \mathrm{~m})$ apresentou anéis de crescimento mais largos, com uma maior proporção de lenho tardio, o que contribuiu para a maior densidade. Esses resultados corroboram os do estudo realizado por Lima et al. (2011) sobre o efeito do espaçamento de plantio na estrutura anatômica da madeira de teca. Esses autores observaram que a madeira do maior espaçamento apresentou fibras mais longas e com paredes mais espessas e menor frequência de vasos, resultando em madeiras de maior densidade.

A madeira de cerne e de alburno apresentaram diferenças significativas de densidade nos dois espaçamentos. No maior espaçamento $(6 \times 2 \mathrm{~m})$, o alburno apresentou maior densidade que o cerne, enquanto que no menor espaçamento $(5 \times 2 \mathrm{~m})$ o comportamento foi inverso. Souza (2010) determinou as dimensões das fibras da madeira de teca com 12 anos de idade na direção medula-casca e observou que as fibras próximas à casca apresentaram maior comprimento e maior espessura de parede, o que pode explicar o resultado encontrado em nosso estudo para o maior espaçamento.

As análises estatísticas mostraram que o espaçamento afetou a cor da madeira de teca (Tabela 1). Para a luminosidade $\left(L^{*}\right)$, a madeira de cerne na seção radial (Figura 1a) não apresentou diferenças significativas entre os dois espaçamentos, entretanto na seção tangencial a madeira de cerne do maior espaçamento $(6 \times 2 \mathrm{~m})$ mostrou-se mais escura (menor valor de $L^{*}$ ) em relação à madeira do menor espaçamento $(5 \times 2 \mathrm{~m})$, como mostra a Figura 1c. Já para a luminosidade da madeira de alburno observaram-se diferenças significativas entre os espaçamentos somente na face radial. Nesse caso, a madeira de alburno do maior espaçamento é mais escura que aquela do menor espaçamento (Figura 1b).

As análises estatísticas também indicaram diferenças significativas entre os espaçamentos para a tonalidade vermelha $\left(\mathrm{a}^{*}\right)$. Tanto na face radial quanto na tangencial, a madeira de cerne do maior espaçamento apresentou mais pigmento vermelho (maior valor de $\mathrm{a}^{\star}$ ) que aquela do menor espaçamento (Figuras 1a e 1c). Na seção radial, a madeira de alburno também apresentou mais pigmento vermelho no maior espaçamento (Figura $1 \mathrm{~b}$ ). Entretanto, na seção tangencial, o espaçamento não afetou a coordenada $\mathrm{a}^{\star}$ da madeira de alburno (Figura 1d). 

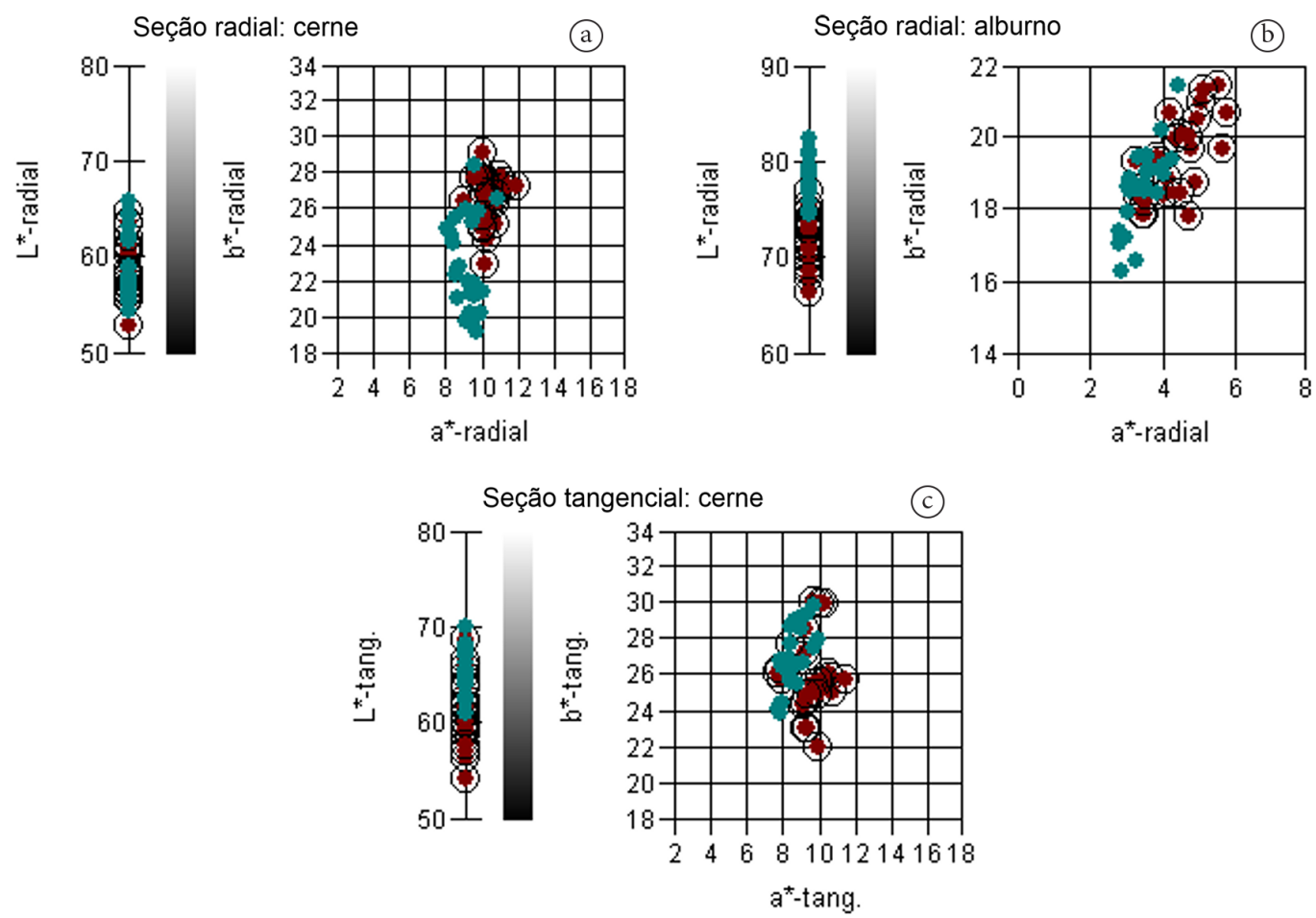

Espaçamento $6 \times 2 \mathrm{~m}$ Espaçamento $5 \times 2 \mathrm{~m}$

Figura 1. Coordenadas colorimétricas da madeira de teca proveniente de dois diferentes espaçamentos. (a,b) seção radial das madeiras de cerne e alburno, respectivamente; (c) seção tangencial das madeiras de cerne e alburno, respectivamente.

Figure 1. Color coordinates of the teak wood from two different spacing. (a, b) radial section of the heartwood and sapwood, respectively; (c) tangential section of the hartwood and sapwood, respectively.

A madeira de cerne do maior espaçamento também apresentou mais tonalidade amarela $\left(b^{\star}\right)$ na face radial (Figura 1a). Entretanto, o mesmo não ocorreu na face tangencial, onde não foram encontradas diferenças significativas entre os espaçamentos (Figura 1c). Para o alburno não foram observadas diferenças significativas entre os espaçamentos para a variável $b^{*}$ em nenhuma das seções, tanto radial quanto tangencial.

Os resultados obtidos para as coordenadas colorimétricas $\mathrm{L}^{\star}, \mathrm{a}^{*}$ e $\mathrm{b}^{\star}$ mostram que o espaçamento afeta a cor da madeira de teca, sendo que, no maior espaçamento, a madeira de cerne é mais escura e apresenta mais pigmento vermelho e mais pigmento amarelo. A madeira de alburno do maior espaçamento também é mais escura e apresenta mais pigmento vermelho na face radial. Segundo alguns autores (Rink, 1987; Gonçalez, 1993), a cor natural da madeira pode ser afetada por: características físicas (ex.: densidade e teor de umidade), estrutura anatômica (ex.: anéis de crescimento, vasos, raios e tipo de parênquima axila), teor de extrativos, tratos silviculturais, taxa de crescimento, altura, diâmetro e idade da árvore, condições edáficas e climáticas.

\subsection{Efeito do espaçamento na variação do teor de extrativos da madeira}

As madeiras de cerne e alburno do maior espaçamento $(6 \times 2 \mathrm{~m})$ apresentaram os maiores teores de extrativos totais (4,35 e 1,55\%, respectivamente) quando comparadas àquelas do menor espaçamento, cujos teores foram de 3,35 e 1,20\% para cerne e alburno, respectivamente (Tabela 2). Tal como esperado, o cerne apresentou maior teor de extrativos que o alburno, conforme mencionado por Desch \& Dinwoodie (1993). Segundo o Forest Products Laboratory (FPL, 2010), a cor do cerne depende da presença, características e teor dos extrativos na madeira. Os extrativos também podem afetar a densidade da madeira e são responsáveis 
pela durabilidade natural e resistência à degradação fúngica. No caso da madeira de teca, a estabilidade dimensional e a resistência à água são propriedades conferidas em parte pela presença de ceras e óleos formados e depositados no cerne. Quanto à relação densidade/extrativos, Oliveira (2011) encontrou diferenças significativas entre a densidade da madeira de teca com extrativos e a densidade da madeira de teca sem extrativos, valores médios de 0,669 e 0,631 g. $\mathrm{cm}^{-3}$, respectivamente.

\subsection{Efeito da densidade na variação da cor da madeira}

As análises de correlação entre a densidade e as coordenadas de cor indicaram correlações significativas, tanto para o cerne quanto para o alburno (Tabela 3). A densidade do cerne apresentou uma correlação positiva $(r=0,8509)$ com a coordenada $\mathrm{b}^{\star}$ na face radial (Figura 2a), indicando que as madeiras mais densas do cerne apresentam mais pigmento amarelo na seção

Tabela 2. Teor de extrativos da madeira de teca proveniente de diferentes espaçamentos.

Table 2. Extractive content of the teak wood from two different spacing.

\begin{tabular}{cccccc}
\multirow{2}{*}{ Teor de extrativos (\%) } & \multicolumn{2}{c}{ Espaçamento $\mathbf{6} \times \mathbf{2} \mathbf{~ m}$} & & \multicolumn{2}{c}{ Espaçamento $\mathbf{5} \times \mathbf{2} \mathbf{~ m}$} \\
\cline { 2 - 3 } \cline { 5 - 6 } & Cerne & Alburno & & Cerne & 0,25 \\
Ciclohexano & 1,10 & 0,40 & & 0,95 & 0,40 \\
Acetato de etila & 1,70 & 0,65 & & 1,25 & 0,55 \\
Metanol & 1,55 & 0,50 & & 1,15 & 1,20 \\
\hline Total & 4,35 & 1,55 & 3,35 & \\
\hline
\end{tabular}
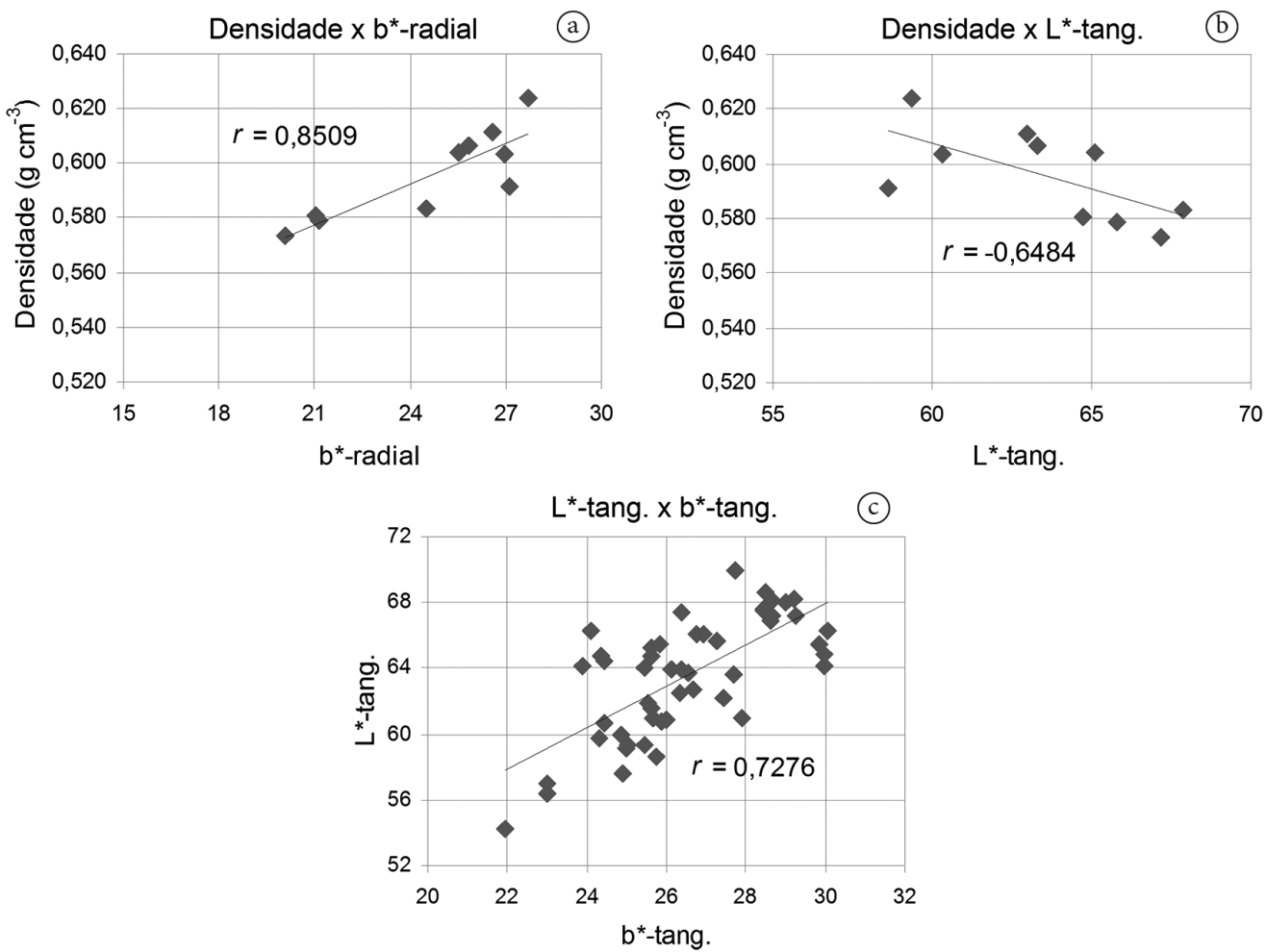

Figura 2. Correlações entre densidade e coordenadas colorimétricas da madeira do cerne de teca. (a) correlação entre densidade e $b^{*}$ na seção radial; (b) correlação entre densidade e $L^{*}$ na seção tangencial; (c) correlação entre as coordenadas $\mathrm{L}^{\star}$ e $\mathrm{b}^{\star}$ na seção tangencial.

Figure 2. Correlation between density and color coordinates of the heartwood of teak. (a) correlation between density and $\mathrm{b}^{\star}$ on radial section; (b) correlation between density and $\mathrm{L}^{*}$ on tangencial section; (c) correlation between $\mathrm{L}^{*}$ and $\mathrm{b}^{*}$ coordinates on tangential section. 
radial. A densidade do cerne também apresentou uma correlação negativa $(r=-0,6484)$ com a coordenada $L^{*}$ na face tangencial (Figura $2 b$ ), enquanto que $L^{*}$-tang e $b^{*}$ tang apresentaram correlação positiva $(r=0,7276)$ (Figura 2c). Esses resultados indicam que, na seção tangencial, as madeiras mais densas do cerne são mais escuras (menor $\mathrm{L}^{\star}$ ) e apresentam menos pigmento amarelo $\left(b^{\star}\right)$. Os diferentes comportamentos de $b^{*}$ nas faces radial e tangencial podem ser explicados pela maior e menor exposição das células de parênquima radial, respectivamente, as quais apresentam acúmulos de extrativos no lúmen. Esses resultados estão de acordo com estudos realizados pelo Forest Products Laboratory (FPL, 2010), o qual afirma que as madeiras mais densas apresentam maior teor de extrativos e são mais escuras.

A densidade da madeira de alburno apresentou uma correlação negativa com a coordenada $L^{\star}$ tanto na face radial quanto na tangencial, com coeficientes de $-0,8545$ e $-0,7161$, respectivamente (Figuras 3a e 3d).
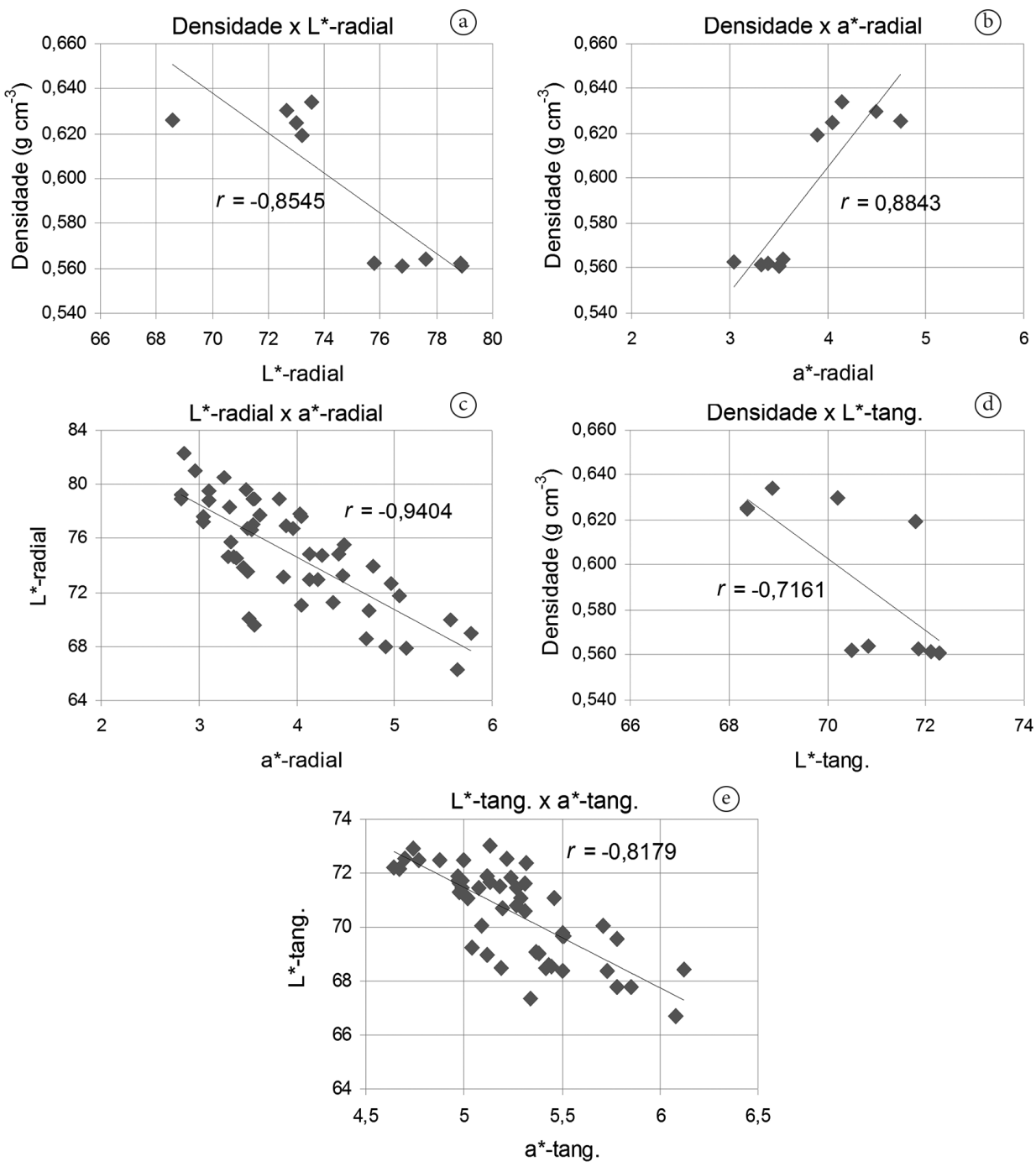

Figura 3. Correlações entre densidade e coordenadas colorimétricas da madeira do alburno de teca. ( $a, b)$ : correlações entre densidade e as coordenadas $\mathrm{L}^{*} \mathrm{e} \mathrm{a}^{\star}$ na seção radial; $(c, e)$ correlações entre as coordenadas $\mathrm{L}^{*} \mathrm{e} \mathrm{a}^{*}$ nas seções radial e tangencial, respectivamente; $(d)$ correlação entre densidade e $L^{\star}$ na seção tangencial.

Figure 3. Correlation between density and color coordinates of the sapwood of teak. $(\mathrm{a}, \mathrm{b})$ correlations between density and $\mathrm{L}^{\star}$ and $\mathrm{a}^{\star}$ coordinates on radial section; (c, e) correlations between $\mathrm{L}^{\star}$ and $\mathrm{a}^{\star}$ coordinates on radial and tangential sections, respectively; (d) correlation between density and $\mathrm{L}^{\star}$ on tangential section. 
Uma correlação positiva $(r=0,8843)$ também foi observada entre a densidade e a tonalidade vermelha $\left(\mathrm{a}^{*}\right)$ na face radial (Figura $3 \mathrm{~b}$ ). As coordenadas $\mathrm{L}^{\star} \mathrm{e}$ $\mathrm{a}^{\star}$ apresentaram uma correlação negativa tanto na face radial quanto na tangencial, com coeficientes de $-0,9404$ e $-0,8179$, respectivamente (Figuras $3 c$ e $3 \mathrm{e}$ ). Esses resultados mostram que as madeiras mais densas de alburno são mais escuras e apresentam mais pigmento vermelho $\left(\mathrm{a}^{*}\right)$.

Para as análises de correlação, considerando todos os dados (cerne + alburno), foram observadas correlações negativas entre $L^{*} e$ as variáveis cromáticas $\left(a^{*}\right.$ e $\left.b^{*}\right)$, para ambas as seções, enquanto que as coordenadas $\mathrm{a}^{\star} \mathrm{e} \mathrm{b}^{\star}$ apresentaram uma correlação positiva. Isso mostra que a madeira do alburno, mais clara (maior $\mathrm{L}^{\star}$ ), apresenta menos pigmento vermelho $\left(a^{*}\right)$ e menos pigmento amarelo $\left(b^{*}\right)$, enquanto que a madeira do cerne, mais escura (menor $\mathrm{L}^{*}$ ) apresenta mais pigmento vermelho e mais pigmento amarelo.

\subsection{Efeito do teor de extrativos na variação da cor da madeira}

Para as análises de correlação entre os extrativos e as coordenadas de cor foram considerados todos os dados, incluindo cerne e alburno. Foram observadas correlações significativas entre o teor de extrativos total e as coordenadas $\mathrm{L}^{*}$ e $\mathrm{a}^{*}$ para ambas as seções (Tabela 3). O teor de extrativos apresentou uma correlação negativa com a coordenada $\mathrm{L}^{\star}$ tanto na

Tabela 3. Análises de correlação de Pearson entre densidade, teor de extrativos e coordenadas colorimétricas da madeira de cerne e de alburno de teca.

Table 3. Pearson's correlation between density, extractive content and color coordinates of the heartwood and sapwood of teak.

\begin{tabular}{|c|c|c|c|c|c|c|}
\hline \multicolumn{7}{|c|}{ Seção radial } \\
\hline & & Densidade & Extrativos & $\mathbf{L}^{\star}$-radial & $a^{*}$-radial & $b^{*}$-radial \\
\hline \multirow{4}{*}{ Cerne } & Densidade & 1 & & & & \\
\hline & $\mathrm{L}^{*}$-radial & 0,2783 & - & 1 & & \\
\hline & $\mathrm{a}^{\star}$-radial & 0,3731 & - & $-0,2805$ & 1 & \\
\hline & $\mathrm{b}^{*}$-radial & $0,8509^{* *}$ & - & 0,4216 & 0,5284 & 1 \\
\hline \multirow{4}{*}{ Alburno } & Densidade & 1 & & & & \\
\hline & $L^{\star}$-radial & $-0,8545^{\star *}$ & - & 1 & & \\
\hline & $a^{*}$-radial & $0,8843^{\star *}$ & - & $-0,9404^{\star *}$ & 1 & \\
\hline & $\mathrm{b}^{*}$-radial & 0,3940 & - & $-0,4057$ & 0,5560 & 1 \\
\hline \multirow{5}{*}{ Cerne + alburno } & Densidade & 1 & & & & \\
\hline & Extrativos & 0,2078 & 1 & & & \\
\hline & $\mathrm{L}^{\star}$-radial & $-0,1960$ & $-0,9558^{\star}$ & 1 & & \\
\hline & $a^{*}$-radial & 0,1434 & $0,9900^{* *}$ & $-0,9553^{* *}$ & 1 & \\
\hline & $\mathrm{b}^{*}$-radial & 0,2758 & 0,9470 & $-0,7365^{\star \star}$ & $0,8676^{\star *}$ & 1 \\
\hline \multicolumn{7}{|c|}{ Seção tangencial } \\
\hline & & Densidade & Extrativos & $L^{*}$-tang & $a^{*}$-tang & $b^{*}$-tang \\
\hline \multirow{4}{*}{ Cerne } & Densidade & 1 & & & & \\
\hline & $L^{*}-\operatorname{tang}$ & $-0,6484^{\star}$ & - & 1 & & \\
\hline & $\mathrm{a}^{*}$-tang & 0,4007 & - & $-0,4856$ & 1 & \\
\hline & $b^{\star}-\tan g$ & $-0,1888$ & - & $0,7276^{*}$ & 0,0523 & 1 \\
\hline \multirow{4}{*}{ Alburno } & Densidade & 1 & & & & \\
\hline & $L^{\star}-\operatorname{tang}$ & $-0,7161^{\star}$ & - & 1 & & \\
\hline & $a^{*}$-tang & 0,4662 & - & $-0,8179^{\star *}$ & 1 & \\
\hline & $b^{\star}-\tan g$ & $-0,5862$ & - & 0,1230 & 0,1951 & 1 \\
\hline \multirow{5}{*}{ Cerne + alburno } & Densidade & 1 & & & & \\
\hline & Extrativos & 0,2078 & 1 & & & \\
\hline & $L^{*}$-tang & $-0,3186$ & $-0,9854^{*}$ & 1 & & \\
\hline & $a^{\star}-\operatorname{tang}$ & 0,1009 & $0,9909^{\star \star}$ & $-0,8732^{\star \star}$ & 1 & \\
\hline & $\mathrm{b}^{*}-\operatorname{tang}$ & $-0,0811$ & 0,8967 & $-0,5888^{* *}$ & $0,8711^{* *}$ & 1 \\
\hline
\end{tabular}

${ }^{*}$ Significativo a $95 \%$ de probabilidade. ${ }^{* *}$ Significativo a $99 \%$ de probabilidade. 

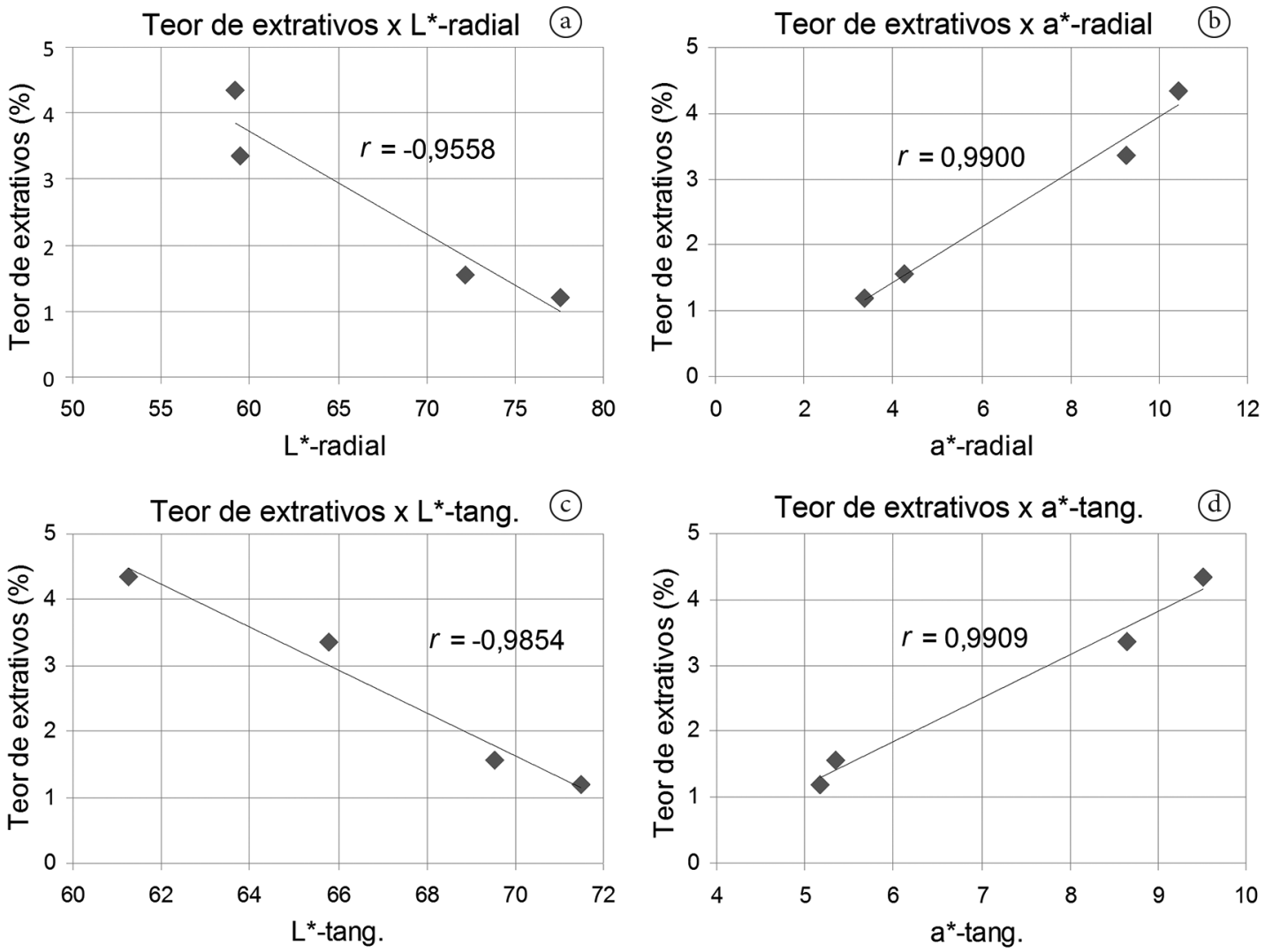

Figura 4. Correlações entre teor de extrativos e coordenadas colorimétricas da madeira de teca. (a, b) correlação entre teor de extrativo e as coordenadas $L^{\star}$ e $a^{\star}$ na seção radial; (c, d) correlação entre teor de extrativos e as coordenadas $\mathrm{L}^{*}$ e $\mathrm{a}^{*}$ na seção tangencial.

Figure 4. Correlation between extractive content and color coordinates of teak wood. $(a, b)$ correlation between extractive content and $\mathrm{L}^{\star}$ and $\mathrm{a}^{\star}$ coordinates on radial section; $(c, d)$ correlation between extractive content and $\mathrm{L}^{\star}$ and $\mathrm{a}^{\star}$ coordinates on tangential section.

face radial quanto na tangencial, com coeficientes de $-0,9558$ e -0,9854, respectivamente (Figuras 4a e 4c). Uma alta correlação positiva também foi observada entre o teor de extrativos e a variável a ${ }^{\star}$ para ambas as faces, radial e tangencial (Figuras 4 b e $4 d$ ). Esses resultados indicam que as madeiras mais escuras (menor $\mathrm{L}^{*}$ ) apresentam mais pigmento vermelho e mais extrativos, o que caracteriza a madeira de cerne. Esses resultados corroboram os encontrados por Gierlinger et al. (2004). Esses autores também observaram que a cor vermelha $\left(\mathrm{a}^{\star}\right)$ e a luminosidade $\left(\mathrm{L}^{\star}\right)$ da madeira de Larix decidua Mill estava correlacionada com o teor de extrativos.

Estudos realizados por Haupt et al. (2003) mostraram que o teor de extrativos da madeira de teca aumenta com a idade da árvore e que a presença de substâncias do tipo tectoquinona está relacionada à maior durabilidade natural. Gierlinger et al. (2004) também observaram que as madeiras com mais pigmento vermelho $\left(\mathrm{a}^{*}\right)$ apresentam maior resistência à podridão. Sendo assim, como sugerido por Kokutse et al. (2006), a colorimetria apresenta potencial para ser utilizada como indicador de qualidade e durabilidade da madeira, além de apresentar a vantagem de ser um método relativamente barato, simples e portátil.

\section{CONCLUSÕES}

A madeira de teca proveniente do maior espaçamento de plantio apresentou maior densidade e maior teor de extrativos.

Na seção radial, as madeiras de cerne com maior densidade apresentaram mais pigmento amarelo $\left(b^{*}\right)$, enquanto que, na seção tangencial, elas apresentaram- 
se mais escuras (menor $\mathrm{L}^{*}$ ) e com menos pigmento amarelo.

As madeiras de alburno de maior densidade mostraram-se mais escuras (menor $L^{*}$ ) e apresentam mais pigmento vermelho $\left(\mathrm{a}^{*}\right)$, tanto na seção radial quanto na tangencial.

As madeiras mais escuras (menor $\mathrm{L}^{*}$ ) e com mais pigmento vermelho $\left(\mathrm{a}^{\star}\right)$ apresentaram um maior teor de extrativos.

As coordenadas de cor variaram de acordo com o plano de corte observado, indicando que a orientação e/ou proporção das células afeta a cor da madeira.

A colorimetria pode ser utilizada como uma ferramenta na classificação e no controle de qualidade das peças de madeira de teca em função das exigências do mercado.

\section{STATUS DA SUBMISSÃO}

Recebido: 22 mar., 2013

Aceito: 24 out., 2015

\section{AUTOR(ES) PARA CORRESPONDÊNCIA}

\section{Rosilei Aparecida Garcia}

Departamento de Produtos Florestais, Universidade Federal Rural do Rio de Janeiro UFRRJ, Rodovia BR 465, Km 07, CEP 23897-000, Seropédica, RJ, Brasil

e-mail: rosileigar@hotmail.com

\section{REFERENNCIAS}

Abreu HS, Carvalho AM, Monteiro MBO, Pereira RPW, Silva HR, Souza KCA et al. Métodos de análise em química da madeira. Seropédica: UFRRJ; 2006. 20 p. Série Técnica. Floresta e Ambiente.

American Society for Testing and Materials - ASTM. D2395-93: standard test methods for specific gravity of wood and wood-based materials. Philadelphia: ASTM; 1999a.

American Society for Testing and Materials - ASTM. D1105-96: standard test method for preparation of extractivefree wood. Philadelphia: ASTM; $1999 \mathrm{~b}$.

Associação Brasileira Técnica de Celulose e Papel - ABTCP. Normas técnicas. São Paulo: ABTCP; 1971.

Baillères H, Durand PY. Non destructive techniques for wood quality assessment of plantation-grown teak. Bois et Forêts des Tropiques 2000; 283: 17-27.
Derkyi NSA, Baillères H, Chaix G, Thevenom MF, Oteng-Amoako AA, Adu-Bredu S. Colour variation in teak (Tectona grandis) wood from plantations across the ecological zones of Ghana. Ghana Journal of Forestry 2009; 25: 40-49.

Desch HE, Dinwoodie JM. Timber-its structure, properties and utilization. London: McMillan; 1993.

Forest Products Laboratory - FPL. Wood handbook: wood as an engineering material. Madison: U.S. Department of Agriculture, Forest Service, Forest Products Laboratory; 2010. 508 p.

Gierlinger N, Jacques D, Grabner M, Wimmer R, Schwanninger M, Rozenberg $\mathrm{P}$ et al. Colour of larch heartwood and relationships to extractives and brown-rot decay resistance. Trees: Structure and Function 2004; 18(1): 102-108. http://dx.doi.org/10.1007/s00468-003-0290-y.

Gonçalez JC. Caractérisation technologique de quatre espèces peu connues de la forêt amazonienne: anatomie, chimie, couleur, propriétés physiques et mécaniques [tese]. Nancy: École Nationale de Génie Rural, des Eaux et des Forêts, Université de Nancy I; 1993.

Haupt M, Leithoff D, Meier D, Puls J, Richter HG, Faix O. Heartwood extractives and natural durability of plantation grown teakwood (Tectona grandis L.): a case study. Holz als Roh- und Werkstoff2003; 61(6): 473-474. http://dx.doi. org/10.1007/s00107-003-0428-Z.

International Organization for Standardization - ISO. ISO 11664-4:2008: colorimetry: part 4: CIE $1976 L^{*} a^{\star} b^{*}$ : colour space. Geneva: ISO; 2008.

Janin G, Gonçalez J, Ananias R, Charrier B, Fernandes G, Dilem A. Aesthetics appreciation of wood colour and patterns by colorimetry. Part 1. Colorimetry theory for the CIELab System. Maderas: Ciencia y Tecnología 2001; 3: 3-13.

Kokutse AD, Stokes A, Baillères H, Kokou K, Baudasse C. Decay resistance of Togolese teak (Tectona grandis L. f.) heartwood and relationship with colour. Trees 2006; 20(2): 219-223. http://dx.doi.org/10.1007/s00468-005-0028-0.

Konica Minolta. Comunicação precisa da cor: controle de qualidade da percepção à instrumentação. Seoul: Konica Minolta; 1998.

Lima IL, Garcia R, Longui EL, Florsheim SMB. Dimensões anatômicas da madeira de Tectona grandis Linn. Em função do espaçamento e da posição radial do tronco. Scientia Forestalis 2011; 39(89): 61-68.

McGinnes EA, Phelps JE. Growth-quality evaluation of black walnut wood. Part III: An anatomical study of color characteristics of black walnut veneer. Wood and Fiber Science 1983; 15(3): 212-218.

Mady FTM. Conhecendo a madeira: informações sobre 90 espécies comerciais. Manaus: SEBRAE/AM/Programa de Desenvolvimento Empresarial e Tecnológico; 2000. 
Miranda I, Sousa V, Pereira H. Wood properties of teak (Tectona grandis) from a mature unmanaged stand in East Timor. Journal of Wood Science 2011; 57(3): 171-178. http://dx.doi.org/10.1007/s10086-010-1164-8.

Mori CLSO, Lima JT, Mori FA, Trugilho PF, Goncalez JC. Caracterização da cor da madeira de clones de híbridos de Eucalyptus spp. Cerne 2005; 11(2): 137-146.

Mori CLSO, Mori FA, Lima JT, Trugilho PF, Oliveira AC. Influência das características da cor da madeira de eucaliptos. Ciência Florestal 2004; 14(2): 123-132.

Möttönen K, Alvila L, Pakkanen T. CIELab Measurements to determine the role of felling season, log storage and kiln drying on coloration of silver birch wood. Scandinavian Journal of Forest Research 2002; 17(2): 179-191. http:// dx.doi.org/10.1080/028275802753626827.

Moya R, Pérez D. Processing and marketing of teakwood products from fast-grown teak plantations in Costa Rica. In: Proceedings of Regional Workshop Products and Marketing of Teak Wood Products of Planted Forest; 2007; Peechi. India: KFRI; 2007. p. 24-32.

Narayanamurti D, Singh J. Caoutchouc in Teak. Journal Composite Wood 1960; 7(4): 39-41.

Oliveira BRU. Dendrocronologia e análise da variação radial da densidade do lenho de árvores de Tectona grandis L. f., do município de Cáceres, MT [dissertação]. Seropédica: Universidade Federal Rural do Rio de Janeiro; 2011.
Panshin AJ, Zeeuw C. Textbook of wood technology. 4. ed. New York: McGraw-Hill; 1980.

Premrasmi T, Dietrichs HH. Nature and distribution of extractives in teak (Tectona grandis Linn.) from Thailand. The Natural History Bulletin of Siam Society 1967; 22(12): $1-14$

Rink G. Heartwood color and quantity variation in a Young Black walnut progeny test. Wood and Fiber Science 1987; 15(3): 93-100.

Schmincke KH. Plantaciones de teca en Costa Rica: la experiencia de la empresa Precious Woods. Unasylva 2000; 201: 1-8

Souza MT. Influência do espaçamento e da posição radial nas dimensões das fibras da madeira de Tectona grandis Linn. f. [monografia]. Seropédica: Universidade Federal Rural do Rio de Janeiro; 2010.

Tsoumis G. Wood as raw material: source, structure, chemical composition, growth, degradation, and identification. Oxford: Pergamon Press; 1968.

Yamamoto K, Simatupang MH, Hashim R. Caoutchouc in teak wood (Tectona grandis L. f.): formation, location, influence on sunlight irradiation, hydrophobicity and decay resistance. Holz als Roh- und Werkstoff 1998; 56(3): 201-209. http://dx.doi.org/10.1007/s001070050299. 